\title{
MERS coronavirus: current status
}

\author{
Abbreviations: CDC, Centers for Disease Control; MERS- \\ CoV, Middle East Respiratory Syndrome Coronavirus; RT-PCR, \\ Reverse Transcriptase Polymerase Chain Reaction; VLP, Virus Like \\ Particles
}

Recently, a new virus started to infect certain individuals in the Middle-East. It was soon identified as a previously unknown coronavirus that caused severe respiratory disease with a high rate of mortality. This virus, MERS-CoV, is still closely watched by health authorities as it has the potential to evolve and cause a major epidemic.

In early May 2014, the Centers for Disease Control and Prevention (CDC) announced the first case of Middle East Respiratory Syndrome coronavirus (MERS-CoV) infection in the USA. Reportedly, the patient flew from Saudi Arabia to the United Kingdom (UK) and then to Chicago on April 24, before travelling by bus to Indiana. The patient started experiencing respiratory symptoms on April 27, according to the $\mathrm{CDC}$, and laboratory tests confirmed that he was infected with the MERS-CoV.

MERS-CoV started getting scientists' and media attention in June 2012, when a 60-year old Saudi man was admitted to a private hospital in Jeddah, Saudi Arabia, with a 7-day history of fever, cough, expectoration, and shortness of breath. ${ }^{1}$ On day 11 after admission, the patient died of progressive respiratory and renal failure. Nucleic acids were extracted from sputum samples obtained from the patient's lungs and infected cell cultures at the Erasmus University Medical Centre in the Netherlands and tested by reverse-transcriptase-polymerasechain-reaction (RT-PCR) assays, which revealed that the patient was infected by a novel coronavirus that became known as MERS-CoV. ${ }^{2}$

In September of the same year, the World Health Organization (WHO) was notified by UK health authorities of an individual with a history of travel to Saudi Arabia and Qatar, showing symptoms of acute respiratory syndrome and renal failure after being transferred from a Qatar hospital to the UK. This patient, a 49 year-old male Qatari national, was diagnosed with MERS-CoV infection after comparing the sequence of his virus isolate with that of the virus previously sequenced at the Erasmus University Medical Centre.

As of May 2014, MERS-CoV has been detected in Saudi Arabia, Qatar, Jordan, the United Arab Emirates (UAE), Oman, Kuwait, Yemen, Lebanon, Iran, Algeria, the UK, France, Italy, Greece, Germany, the Netherlands, Tunisia, Egypt, Malaysia, the Philippines and the USA. So far, the WHO has reported 701 laboratory-confirmed human cases, including at least 249 related deaths (about 35\% mortality based on these figures). MERS-CoV causes sudden and serious respiratory illness with fever, cough, and shortness of breath and breathing difficulties, especially in persons with underlying medical conditions and in older individuals. At present there is no evidence for sustained community transmission, however there is a serious possibility that the virus could evolves and adapt towards efficient human-to-human transmission. The virus is believed to have an animal host as natural reservoir but although some camels have been found to harbour antibodies to the virus, it is not clear at this point what is their role, if any, in virus transmission.

Coronaviridae represents a family of enveloped viruses harbouring large club-shaped surface projections which are very characteristic
Volume I Issue 2 - 2014

\author{
Jocelyn Yelle \\ Antiviral InteliStrat Inc., Canada
}

Correspondence: Jocelyn Yelle, Antiviral InteliStrat Inc., 3077 Edouard-Montpetit St., Suite 406, Laval, Quebec, Canada, Tel I-5 |4-8|6-8856, Fax |-5|4-227-53|5,

Email yellej@videotron.ca

Received: June 21, 2014 | Published: July 01, 2014

in electron micrographs and are responsible for the name "coronavirus". Coronaviruses have an exceptionally large, positive-sense RNA genome. In the particular case of MERS-CoV, it is 30,119 nucleotides in length. ${ }^{3}$ Importantly, MERS-CoV is distinct from the SARS-CoV that caused the first coronavirus-associated epidemic in humans in $2002,{ }^{4-6}$ although it produces similar clinical symptoms in infected individuals. MERS-CoV is also distinct from the commoncold coronavirus and known endemic human coronaviruses $\mathrm{HCoV}$ OC43 and HCoV-HKU1. ${ }^{2,3}$

Research on prevention and treatment of coronavirus infection has been relatively neglected before 2002 as these viruses usually caused only mild respiratory infections in healthy adults. After the start of the SARS-CoV epidemic that affected several thousand people in 29 countries and killed about $9 \%$ of those infected, the situation changed drastically. About 25 different groups in various countries, both in academic research centers and in companies, quickly announced officially they were working on SARS vaccines, immunomodulators or antibodies, while at least 15 more started evaluating all sorts of molecules potentially acting as inhibitors for the virus (Source: Antiviral InteliStrat's database). Most of these candidate vaccines and drugs were not developed beyond preclinical stage, with a few exceptions. A DNA-based vaccine developed by the Vaccine Research Center of the NIAID/NIH and manufactured by Vical went successfully through a Phase I trial, showing safety and eliciting neutralizing antibodies in $80 \%$ ( 8 out of 10 ) of volunteers who received the vaccine. Also, a Chinese firm (Sinovac Biotech) completed a Phase I trial with a SARS vaccine consisting of inactivated whole virions, with no safety issues 
and 24 of 36 vaccine recipients developing neutralizing antibodies. Besides vaccination, a combination of Lopinavir and Ritonavir, two protease inhibitors approved for HIV/AIDS treatment, under the trade name Kaletra ${ }^{\mathrm{TM}}$, along with Ribavirin showed some efficacy in a Phase I/II trial in Hong Kong. However, as the menace of SARS$\mathrm{CoV}$ vanished in 2003, the vast majority of these efforts stopped. Nevertheless, this constituted an important foundation for the new battle against MERS-CoV.

In the case of MERS-CoV, so far only a few potential vaccines and drug candidates, all at very early stages of development, have been announced or published, mostly because of the relatively small number of infected people and the current perception of a low risk of epidemic. The total cost of development of a vaccine or antiviral drug remains considerable and needs to be taken into account before launching such a program.

On the prevention side, at least one candidate vaccine against MERS-CoV has been announced by Novavax, a US company. The vaccine consists in virus-like particles (VLP) obtained using the firm's proprietary recombinant nanoparticle technology, with MERS-CoV's major surface protein $\mathrm{S}$, which is responsible for viral attachment to target cells, exposed on the surface of the VLPs. The vaccine, which also contains a proprietary adjuvant called Matrix- $\mathrm{M}^{\mathrm{TM}}$, is currently being tested in animals, with some data recently published. ${ }^{7}$ Despite these promising results, there is an important question that will need to be answered before a MERS-CoV vaccine reaches the market: who will need to be vaccinated? As long as the source of infection is not clearly identified and the number of infected people remains limited, it is difficult to determine if the development of a vaccine is justified and financially viable.

On the therapeutic side, a few groups have recently published some data on compounds showing inhibitory properties against MERS-CoV. Some of these are briefly listed here. First, de Wilde et al. screened a library of 348 FDA-approved drugs for anti-MERS-CoV activity in cell culture. ${ }^{8}$ They identified four compounds (chloroquine, chlorpromazine, loperamide, and lopinavir) able to inhibit MERS$\mathrm{CoV}$ replication at a $50 \%$ effective concentration (EC50) between 3 and 8 micromolar $(\mu \mathrm{M})$. Interestingly, the compounds also inhibited the replication of SARS-CoV. Another group followed a slightly different approach consisting in the repurposing of existing drugs, and tested a library of 290 compounds selected on the basis of current or previous FDA approval or advanced clinical development. ${ }^{9}$ They identified 27 compounds with various structures and pharmaceutical classes with activity against both MERS-CoV and SARS-CoV. The anti-MERS-CoV activity (EC50) varied from $3 \mathrm{nM}$ (space between 3 and $\mathrm{nM}$ ) for anisomycin, an antibiotic blocking protein synthesis, to $17 \mu \mathrm{M}$ for imatinib mesylate (Gleevec), a tyrosine kinase inhibitor used in oncology. The advantage of screening FDA-approved (or well advanced in the clinic) compounds resides in the fact that these molecules have known properties and in many cases have already gone through the whole approval process compared to novel compounds. On the other hand, the mode of action of these molecules in the particular case of MERS-CoV will need to be elucidated. A more direct approach was taken by Adedeji et al. ${ }^{10,11}$ who recently described a small 1,2,4 triazole derivative compound called SSYA10-001 that inhibited the viral NTPase/helicase (known as nonstructural protein 13, nsp13) of both SARS-CoV and MERS-CoV. The antiviral activity (EC50) in cell cultures was about $25 \mu \mathrm{M}$ for MERS-CoV and $7 \mu \mathrm{M}$ for SARS-CoV, with no significant cytotoxicity at a concentration of $500 \mu \mathrm{M}$. Interestingly, no helicase inhibitors have been approved so far as antiviral agents.
There is still much work to do before one of these compounds, or a derivative of them, become a new drug for treating the infection. The antiviral activity of most of these compounds is relatively low for clinical use. On the other hand, they represent excellent lead molecules for rational drug design of more potent products. In addition, they do inhibit these viruses although in most cases the activity has only been shown in vitro. In case of emergency, some of these compounds could theoretically be used under strict conditions to limit disease progression and stabilize patients.

Although there are yet no vaccines or therapeutic agents for MERS-CoV infection, significant progress has been made towards achieving this goal. If the virus eventually acquires the ability to be transmitted efficiently between humans, we should be better prepared to contain a coronavirus epidemic than we were in 2002 .

\section{Conflict of interest}

The author is President of Antiviral InteliStrat Inc. He declares having no financial links or interests in the other organizations listed in the manuscript.

\section{Acknowledgment}

None.

\section{References}

1. Zaki AM, van Boheemen S, Bestebroer TM, et al. Isolation of a novel coronavirus from a man with Pneumonia in Saudi Arabia. N Engl J Med. 2012;367(19):1814-1820.

2. de Groot RJ, Baker SC, Baric RS, et al. Middle East respiratory syndrome coronavirus (MERS-CoV): Announcement of the Coronavirus Study Group. J Virol. 2013;87(14):7790-7792.

3. van Boheemen S, de Graaf M, Lauber C, et al. Genomic characterization of a newly discovered coronavirus associated with acute respiratory distress syndrome in humans. MBio. 2012;3(6):e00473-12.

4. Ksiazek TG, Erdman D, Goldsmith CS, et al. A novel coronavirus associated with severe acute respiratory syndrome. $N$ Engl $\mathrm{J} \mathrm{Med}$. 2003;348(20):1953-1966.

5. Heymann DL, Rodier G. Global surveillance, national surveillance, and SARS. Emerg Infect Dis. 2004;10(2):173-175.

6. Peiris JS, Lai ST, Poon LL, et al. Coronavirus as a possible cause of severe acute respiratory syndrome. Lancet. 2003;361(9366):1319-1325.

7. Coleman CM, Liu YV, Mu H, et al. Purified coronavirus spike protein nanoparticles induce coronavirus neutralizing antibodies in mice. Vaccine. 2014;32(36):3169-3174.

8. de Wilde AH, Jochmans D, Posthuma CC, et al. Screening of an FDAapproved compound library identifies four small-molecule inhibitors of Middle East respiratory syndrome coronavirus replication in cell culture. Antimicrob Agents Chemother. 2014;pii:AAC.03011-14.

9. Dyall J, Coleman CM, Hart BJ, et al. Repurposing of clinically developed drugs for treatment of Middle East respiratory coronavirus infection. Antimicrob Agents Chemother. 2014;pii:AAC.03036-14.

10. Adedeji AO, Singh K, Calcaterra NE, et al. Severe acute respiratory syndrome coronavirus replication inhibitor that interferes with the nucleic acid unwinding of the viral helicase. Antimicrob Agents Chemother. 2012;56(9):4718-4728.

11. Adedeji AO, Singh K, Kassim A, et al. Evaluation of SSYA10-001 as a replication inhibitor of SARS, MHV and MERS coronaviruses. Antimicrob Agents Chemother. 2014;AAC.02994-14. 